\title{
Schranken und Quadranten
}

\section{Hegemoniale Diskurse und Praktiken zu Inter* und Trans* in der Psychotherapie}

\author{
Lisa Wanner · Finn L. Landsteiner
}

Online publiziert: 28. Mai 2019

(C) Der/die Autor(en) 2019

Zusammenfassung Der Artikel beschäftigt sich mit Fragen zu vielfältigen Geschlechtlichkeiten in psychotherapeutischen Räumen. Wir zeichnen eine Verbindung zwischen Topoi des hegemonialen Diskurses um Inter* und Erfahrungen von Trans* und Gender non Conforming Personen, die Therapie in Anspruch nehmen. Möglichkeiten und Grenzen geschlechtlicher Subjektivierung werden in Diskursen und Praktiken - maßgebend in klinischen - hervorgebracht. Vor diesem Hintergrund diskutieren wir die ambivalente Rolle von Psychotherapeut*innen als Vertreter*innen eines machtstützenden Systems, das gleichzeitig individuelle Selbstbestimmung stärken soll. Aus den Überlegungen leiten wir spezifische Bestärkungsund Gefahrenpotentiale psychotherapeutischer Räume und Reflexionsebenen für Psychotherapeut*innen ab.

Schlüsselwörter Inter* · Trans* . Gender .

Diskursanalyse $\cdot$ Psychotherapie .

Handlungsempfehlungen

\section{Wanner $(\bowtie)$}

Karl Landsteiner Privatuniversität

für Gesundheitswissenschaften,

Dr.-Karl-Dorrek-Straße 30, 3500 Krems an der Donau,

Österreich

lisa.wanner@kl.ac.at

F. L. Landsteiner

Institut für Geschichte, Martin-Luther-Universität

Halle-Wittenberg, Adam-Kuckoff-Straße 15, 06108 Halle

(Saale), Deutschland

finnflames@riseup.net

\section{Barriers and Quadrants \\ Hegemonic Discourses and Practices on Inter* and Trans* in Psychotherapy}

Summary The article deals with questions about various genders in psychotherapeutic rooms. We draw a connection between topoi of the hegemonic discourse around Inter* and experiences of Trans* and Gender Non Conforming People as people who are seeking psychotherapy. Possibilities and limitations of gendered subjectivation are produced in decisively clinical discourses and practices. Against this background, we discuss the ambivalent role of psychotherapists as representatives of a power-supporting system that simultaneously strengthens individual selfdetermination. On the basis of these considerations we derive specific strengthening and endangering potentials from psychotherapeutic spaces and highlight levels of reflection for psychotherapists.

Keywords Inter* - Trans*. Gender · Discourse Analysis · Psychotherapy $\cdot$ Recommendations for Practice

I am suggesting that the unconscious imperatives of gender are in operation in all of us, even as some of us may challenge or resist the imperatives.

Natascha Distiller (2011, S. 16)

Besonders durch den Einfluss sozialer Bewegungen (Frauen*-, Lesben- und Schwulen-, Transgender-, Inter*Bewegung) wurden neue Diskurse etabliert, die widerständige, nonkonforme Verkörperungen von Geschlecht artikulierbar machen (Siročić 2015). So werden etwa Inter* und Trans* Anliegen gegenwärtig auch in der Mehrheitsgesellschaft präsenter. Gleichzeitig durchziehen weiterhin problematische Narrative die Geschlechterdiskurse, deren Durchdringung 
des psychotherapeutischen Raums wir reflektieren wollen. Wie lässt sich ein sensibler und gesellschaftspolitisch reflektierter Kontakt mit inter*- und trans*spezifischem Erleben Therapie in Anspruch nehmender Personen ${ }^{1}$ entwickeln? Wir argumentieren, dass es dafür permanenter Reflexion diskursiver Geflechte und deren Verwobensein mit eigenen Wahrnehmungs- und Handlungsweisen aufseiten der Psychotherapeut*innen bedarf.

Daher werden zunächst eine diskursanalytische Arbeit zum hegemonialen Inter* Diskurs von Lisa Wanner (2015) und blitzlichtartige Überlegungen zum Trans* Diskurs vorgestellt. Von dort wird mithilfe von Studienergebnissen ${ }^{2}$, bei denen die Erfahrungen von Trans* und Gender Non Conforming ${ }^{3}$ Personen im Zentrum stehen, eine Brücke zum therapeutischen Alltag gebaut. Besonders fragen wir danach, inwiefern Inter* und Trans* im Kontext von „mental health“ in Gestalt von Pathologisierung „behandelt“ werden und heben Reflexionsebenen hervor, mit denen Therapeut*innen praktisch und theoretisch konfrontiert sind. Im Brennpunkt unserer Auseinandersetzung steht eine schulenübergreifende, gesellschafts- und disziplinenkritische Perspektive auf Diskurse, welche die psychotherapeutische Arbeit insgesamt (be)trifft. Gewiss ist es dabei von Bedeutung, die der psychotherapeutischen Ausrichtung entsprechenden Konzepte und Anwendungsbereiche einzubeziehen. Eine vertiefende und differenzierte Darstellung spezifischer Implikationen würde jedoch den Rahmen des Beitrags übersteigen. Ausführliche Darstellungen geben Austin et al. (2016) für kognitive und verhaltenstherapeutische Ansätze, Lin Fraser (2009) für tiefenpsychologische Ansätze, Knutson und Koch (2018) für humanistische sowie Mallory et al. (2017) für systemische Ansätze.

Anhaltende Pathologisierungen machen es jenseits der therapeutischen Grundorientierung weiterhin notwendig, die Selbstverständlichkeit $\mathrm{zu}$ betonen, dass Inter* und Trans* keine automatische Indikation

\footnotetext{
${ }^{1}$ In Anlehnung an K. Dücker (unveröff. Abschlussarbeit an der Goethe-Universität Frankfurt a. M.); wird in der Folge als TiAnP abgekürzt.

${ }^{2}$ Mangels vorliegender Forschungsliteratur aus dem deutschsprachigen Raum bedienten wir uns überwiegend der Studienerkenntnisse von Forschenden aus dem angloamerikanischen Bereich, davon überwiegend aus den USA. Die Problematik, dass zu Themen, die sogenannte Minoritäten betreffen, wenig Literatur im jeweiligen Sprach- und (Be-)Handlungsfeld vorliegt, ist nicht neu. Aufgrund der unterschiedlichen Feldbedingungen ist hinsichtlich der direkten Anwendung der Findings auf die österreichische Psychotherapiepraxis zwar Vorsicht geboten, doch haben wir viele Aspekte aufgezeigt, die als Diskurse über mental health, Körperpolitik und Geschlecht über nationale und kulturelle Grenzen hinausgehend wirksam sind.

${ }^{3}$ Gender Non-Conforming (wird im Folgenden mit GNC abgekürzt) ist ein Begriff, der sich auf Menschen bezieht, die nicht den stereotypen sozialen oder kulturellen Erwartungen ihres bei der Geburt zugewiesenen Geschlechts entsprechen.
}

für eine Therapie darstellen: „[g]enders [..] open out into lives that are led through many ways of being and feeling well“ (Corbett 2009, S. 126). Um Personen dahingehend zu stärken, ist es wichtig, Gefahrenpotentiale für Verletzungen, Stigmatisierungen und (Re-)Traumatisierungen zu untersuchen und für psychotherapeutische Räume zu benennen (Puckett und Levitt 2015).

What is said is said, what is said is taught, what is said is done (to people): Diskurse zu Inter* und Trans*

Cut me in quadrants,

leave me in the corner.

Anohni (2009)

Inter*

Der Diskurs zu Inter* ist besonders von der brutalen Praxis geprägt, an Menschen, vor allem Säuglingen und Kindern, deren Geschlechtlichkeit von der medizinischen Schablone nicht als weiblich oder männlich erfasst werden kann, nichtkonsensuelle geschlechtsnormierende Operationen durchzuführen (vgl. Voß 2012). Diese werden häufig als gewaltvoll, traumatisierend und verstümmelnd beschrieben (Völling 2010). Inter* Aktivist*innen fordern seit vielen Jahren ein Verbot und machen auf weitreichende Diskriminierung aufmerksam. Der Deutsche Ethikrat war 2011 von der Deutschen Bundesregierung beauftragt worden, eine Stellungnahme $\mathrm{zu}$ „Intersexualität“ $\mathrm{zu}$ verfassen $^{4}$, mit der sich Lisa Wanner (2015) unter Einbezug der dadurch initiierten Debatte in Form einer Kritischen Diskursanalyse (nach Jäger 2009) beschäftigt hat. Die Analyse ist u. a. von der Irritation ausgegangen, dass der Deutsche Ethikrat ${ }^{5}$ (2012) im Gegensatz $\mathrm{zu}$ einer aktuellen EU-Resolution ${ }^{6} \mathrm{zu}$ keiner klaren Ablehnung geschlechtsnormierender Ein- bzw. Übergriffe gelangt und insgesamt ambivalent bleibt. Grundsätzlich hat die Frage interessiert, wie Geschlecht- bzw. Geschlechtskörper von wem auf welche Weise verhandelt werden. Im Zentrum stand die Feinanalyse eines Kapitels aus der Stellungnahme „Intersexualität“ („Geschlechtszuordnung und Geschlechtsidentität“, Stn., S. 27-34). Um diese zu kontextualisieren, wurde die gesamte Debatte ${ }^{7}$

\footnotetext{
${ }^{4}$ UN-Ausschüsse hatten auf Menschenrechtsverletzungen an Inter* Personen hingewiesen (vgl. Wanner 2015, S. 1).

${ }^{5}$ Die Quelle der Stellungnahme des Deutschen Ethikrates wird in Folge als Stn. abgekürzt.

6 ,The European Parliament [..] [s]strongly condemns sex-normalising treatments and surgery" (Europäisches Parlament 2019). Nach Luan Pertl, Obmensch der Plattform Intersex Österreich, ein „Meilenstein“. (Plattform Intersex 2019).

7 Zwei mehrstündige Anhörungen, zahlreiche online erschienene Artikel inklusive Kommentaren, eine Befragung von inter* Personen, Stellungnahmen von Personen, die als „Sachverstän-
} 
sowie einige Online-Artikel aus bekannten deutschsprachigen Medien ${ }^{8}$ gesichtet und ausschnittsweise analytischen Betrachtungen unterzogen. Zentrale Ergebnisse werden vorgestellt.

\section{Situationsaustrocknung qua Abstraktion}

Besonders über die Feinanalyse lässt sich zeigen, wie verschiedene Mittel eingesetzt werden, um zunehmend vom realen Geschehen zu abstrahieren. Dies passiert aber in einem Diskurs, der gerade aus der Artikulation von erfahrener Gewalt und Traumatisierung hervorgegangen ist (Wanner 2015, S. 69f).

Substantivierung Zunächst zeigt sich die Situationsaustrocknung darin, dass im analysierten Text Handlungen zumeist in Form von Verbalsubstantiven auftauchen, etwa als „Zuordnung“, „Kennzeichnung“, „Maßnahmen“, „Interventionen“ (Stn., S. 27). Diesen Begriffen sind Handlungen inhärent; das Prozesshafte, Bewegte ist aber zum fixierten Substantiv aggregiert (Wanner 2015, S. 72). Auffällig an diesen Verbalsubstantiven ist zudem deren Ordnungsdimension. Es sind Begriffe, die für Ordnung sorgen, sie scheinen davon zu wissen, was „normal“ ist, indem sie dahingehend zuordnen, kennzeichnen, maßnehmen, intervenieren. Besser noch: sie sind stumme Zeug*innen eines bereits gefrorenen (nie stattgefundenen?) Geschehens (S. $70 \mathrm{ff}$ ).

„Problemlösung“ ohne menschliches Zutun „Medizinische Maßnahmen“ (Stn., S. 27), obgleich von Inter* Personen deutlich kritisiert, werden innerhalb des analysierten Textes (diese Tendenzen zeigen sich im gesamten Diskurs) als „Lösung“ (Wanner 2015, S. 111f) der Situation „uneindeutiges Geschlecht“ dargestellt, die als eine Art „Problem“ konstruiert ist (S 109f). Die „medizinische[..] Intervention[..]“ (Stn., S 27), die das „zu behebende Problem“ lösen soll, wird im analysierten Text zur einzigen aktiven und wirkmächtigen Akteur*in - könnte fast sagen hochstilisiert (Wanner 2015, S. 75 f). Hier kündigt sich ein weiteres Charakteristikum der hegemonialen Verhandlung zu Inter* an: die „Menschenleere“ (S. 77). Die Art der rhetorischen Darstellung lässt keinerlei menschliches Zutun vermuten, geschweige denn erkennen. Es ist die „medizinische Intervention[..] “ (Stn., S. 27), die vereindeutigt, ermöglicht, abzielt, beendet, formt, zuordnet (S. 27f). In der auftauchenden sprachlichen Form verlieren die konkreten Erlebnisse an situativer Realität und sind derart reduziert, dass erfahrene Schmerzen in dieser ,abstrakt gewordenen Welt gar nicht denk-, geschweige denn erlebbar scheinen“ (Wanner 2015, S. 77).

dige“ bezeichnet wurden, Pressemitteilungen und schließlich die finale Stellungnahme.

818 Artikel, Zeitraum 2007-12; etwa in Zeit Online, Frankfurter Allgemeine (FAZ).
Entpersonifizierung des Außer-Ordentlichen Es fehlen dann nicht nur die Akteur*innen, die Körper von Menschen beschneiden, sondern auch die Menschen, deren Körper beschnitten werden (Wanner 2015, S. $77 \mathrm{ff}$ ). Im Gestus der Entpersonifizierung ist im Text von „solchen Fällen“, oder „Fällen von DSD“ (Stn., S. 27) die Rede - bei diesen könne „die Bestimmung des Geschlechts [..] jedoch schwierig oder unmöglich sein oder sich später auch als falsch herausstellen“ (ebd.). Besonders bemerkenswert ist, dass wieder eine Person auftaucht, wenn ,in solchen Fällen [..] teilweise medizinische Maßnahmen [..] eingesetzt [werden], um das Geschlecht zu vereindeutigen oder die Zuordnung der Person zu einem Geschlecht zu ermöglichen“ (ebd.). Die Strategie der Entpersonifizierung („Fälle“) ist also dort eingesetzt, wo der Text von schwieriger/unmöglicher Bestimmung des Geschlechts handelt. Erst wo eine Zuordnung möglich ist, wird der Fall (magischerweise) wieder zur Person; es ist die medizinische Maßnahme, die zum „,geschlechtlich eindeutig erkennbaren Menschen“ zurecht(erd)rückt“ (Wanner 2015, S. 90).

Rhetorische Komplexitäts-Geste Der Gestus der Komplexität durchzieht die gesamte Debatte zu Inter* und zeigt sich im Feinanalysetext etwa an verschachtelten Satzkonstruktionen, semantischen Wiederholungen mit subtilen Verschiebungen und Negationen (S. $79 \mathrm{ff}$ ). Ein dadurch entstehender Anschein der Differenziertheit erschwert es zum einen, die nahezu unkenntlich gemachten biologistischen Setzungen zu Geschlecht dennoch aufzuspüren. Zum anderen wird die Situationsaustrocknung vorangetrieben: es erreicht sich eine weitere Distanzierung über die vorgeschützte Komplexität der Sprache, die bei näherem Hinsehen als massive Reduktion entlarvt werden kann, denn die Situation selber verschwindet wieder ein Stück mehr: „es ist alles so komplex, es ist beinahe weg“ (S. 81). Statt differenzierter Darstellung menschlichen Erlebens konsolidiert sich über „äußerst festes, äußerst theoretisches, äußerst äußerliches Gerede“ (S. 82) ein abstraktes Gerüst. Hierzu schreibt Slunecko (2012, S. 563), dass „Situations- bzw. Ereigniswissen“ seit der antiken Aufklärung gegenüber abstraktem Wissen entwertet sei. Herrschaftslogisch macht das Sinn, da sich Situationswissen in seiner Bewegtheit weniger leicht (er)greifen lässt (Wanner 2015, S. 82). Im abstrahierten Gebäude hingegen werden reales Erleben und Situation unsichtbar - die Handlungspraxis entzieht sich aufgrund ihrer scheinbaren Inexistenz damit von vornherein jeglicher Kritik (ebd.).

\section{Ordnung}

Geschlecht ist als zentrale Ordnungskategorie hergestellt: in der vom Deutschen Ethikrat durchgeführten Befragung „Zur Situation intersexueller Menschen“ (Bora 2012) wird auffallend oft nach Geschlecht und dessen Zuordnung gefragt; der Feinanalysetext weist schon im Titel aus, was Programm ist: „Ge- 
schlechtszuordnung und Geschlechtsidentität“ (Stn., S. 27). Durch die proklamierte Zuordnung wird ganz im Sinne der Festschreibung Identität geschaffen; Geschlecht wird häufig als „Merkmal“ (ebd.) oder „Kennzeichnung“ (ebd.) benannt - beides Begriffe mit offensichtlichem Ordnungskonnex (Wanner 2015, S. 83).

Ordnung, das sind die anderen Wird ein Mensch, wie oben gezeigt, über die geschlechtliche `Einordnung` zur Person, passiert dies „durch andere“ (Stn., S. 27). Geschlecht sei „kein eindimensionales Merkmal“ (ebd.), es ist zunächst von der physiologischen Dimension die Rede, dann weiter: „Hinzu kommt die Selbstwahrnehmung der betreffenden Menschen, die sich einem Geschlecht, beiden Geschlechtern oder keinem Geschlecht als zugehörig empfinden, sowie ihre soziale Zuordnung zu einem Geschlecht, das heißt die Einordnung durch andere“ (ebd.). Interessant ist das Auftauchen der Akteurin „andere“, da das Verbalsubstantiv grammatikalisch keiner Akteurin bedürfte. Tritt ein Verbalsubstantiv dennoch mit Akteur*innen auf, verstärkt sich der Eindruck des zu Betonenden, der sich auch durch die einleitende Prädisposition verschärft (Wanner 2015, S. 90f). Die Bedeutsamkeit der Akteur*in „andere“ wird durch weitere rhetorische Mittel, nämlich Satzbau und Redundanz, gesteigert. Der verschachtelte Satz fließt in der finalen „Einordnung durch andere“ zusammen. Zudem ist das Geschilderte als 'wahr` bzw. 'gewiss hergestellt (ebd.). Auch in der oben genannten Befragung (Bora 2012, S. 40) deutet sich Ähnliches an, wenn die Frage nach der eigenen Benennung und Verortung der soffiziellen` und somit svon außen kommenden Zuordnung chronologisch nachgeordnet ist.

Selbstwahrnehmung vs. Zuordnung Die vorige Beobachtung verdichtet sich, wenn die Konstruktion des Verhältnisses zwischen Selbstwahrnehmung und sozialer Zuordnung untersucht wird, was anhand der obig beschriebenen Handlungen geschieht (Selbstwahrnehmung, sich als zugehörig empfinden vs. Zuordnung, Einordnung). Die Selbstwahrnehmung wird über das rückbezügliche Verb sich als (zugehörig) empfinden erläutert - dieses Empfinden (als potenziell flüchtiger Moment) wird den Vorgängen der Zuordnung bzw. Einordnung (zweimalige gewichtig auftretende Verbalsubstantive, die von Beständigkeit und Ordnung erzählen) nebenan gestellt (Wanner 2015, S. 92 ff). Hiermit wird sowohl über das Verb in seiner Handlungsnähe (und dadurch auch größeren Vergänglichkeit), als auch über die inhaltliche besprochene Ladung von ssich empfinden schwächung vorgenommen (ebd.). Dies ist in der Logik dieses Diskurses zu verstehen, in dem Ordnung, Festigkeit, Beständigkeit als zentral erstrebenswerte Dimensionen gelten.
In Corpore Veritas Die Theorie der aufgewerteten sozialen Zuordnung gegenüber der Selbstwahrnehmung spitzt sich schließlich $\mathrm{zu}$, wenn der gesamte Prozess der Einordnung über Eigenschaften, die „auf der genetischen, hormonellen und anatomischen Ebene in Erscheinung [treten] “ (Stn., S. 27), naturalisiert wird. Seine Opulenz erreicht dieser Auftritt durch die plötzliche Präsenz einer dynamisch und materiell handelnden Akteur*in einem beinah durchwegs passivierten Text (Wanner 2015, S. 99). Das Auftreten steht „selbstverständlich im Präsens; nichts relativiert den Tatsachengehalt dieser Aussage“ (S. 100), es bedarf „keiner weiteren Erklärung“ (ebd.), der Vorgang geschieht permanent und uneingeschränkt. Auch der Aufbau des Satzes (kurz, simpel, prägnant) verweist auf dessen zentrale Bedeutsamkeit. Wenn in weiterer Folge Selbstwahrnehmung und soziale Zuordnung auftauchen, so kommen diese hinzu zum ınaturhaften Körper und seinen Eigenschaften (S. 101). Das als naturhaft Vorgestellte tut sich damit als Orientierungspunkt auf: es sind nicht Menschen, sondern Eigenschaften, die hier handeln.

Definitionsmacht Die Definitionsmacht bleibt im Sinne der obigen Naturalisierung in den Händen der Medizin. Medizinische Vorgehensweisen werden etwa in der gesichteten Berichterstattung in Onlinemedien zwar kritisiert (Fux 2010: „Irrwege“, „fatale Behandlungsstrategien“), medizinisches Wissen bleibt aber Statthalter*in der ,Wahrheit « über das Geschlecht (Foucault 2012, S. 9). Dazu passt, wie sogenanntes `Expert*innenwissen` als `legitimes`Sprechen einer (emotionalisierten) `Betroffenheit` gegenübergestellt wird. Die Machtverteilung und die Frage, wer hier sprechen darf, wird durch den Umstand illustriert, dass die Befragung sogenannter sSachverständiger (Deutscher Ethikrat 2011a) im Gegensatz zur Befragung von Inter* Personen (Bora 2012) vollständig im offenen Antwortformat gestaltet ist (Wanner 2015, S. 54). Eklatant wird dies auch an einer Stelle der Anhörung („Zur Situation von Menschen mit Intersexualität in Deutschland“, Deutscher Ethikrat 2011b, S. 68), in der Lucie Veith über erfahrene Traumatisierung und Diskriminierung im Zusammenhang mit dem sozialmedizinischen Versorgungssystem und über die Notwendigkeit, Selbsthilfe zu stärken, spricht, und zu weinen beginnt. Der Moderator versucht, mit einem „Ok, vielen Dank“ (Deutscher Ethikrat 2011c, Minute $1: 21: 28^{9}$ ) das Sprechen zu übernehmen, Lucie Veith bleibt beharrlich (Deutscher Ethikrat 2011c, ab Minute 1:21:32): „Kleinen Moment, das ist sehr wichtig. Wenn ich in die Reha gehe, weil ich am Ende bin, weil ich fertig bin, dann muss ich mich verteidigen. Es gibt keine Rehaeinrichtungen für uns. Es gibt keine Traumatherapien für uns [...]. Lebensqualität ist

9 Es existieren zur der Anhörung sowohl eine Simultanmitschrift (Dt.E. 2011b) als auch ein Audiofile (Dt. E. 2011c); die zitierte Stelle ist nur im Audiofile zu hören. 
eigentlich ein Lacher.“. Der Moderator bedankt sich und meint: „[W]ir müssen uns noch einmal vertiefend sachkundig machen, was mit den einzelnen Gebieten wirklich los ist" (ebd.). So gilt die erlebte und artikulierte Erfahrung offenbar nicht als „wirkliches“ Wissen - es bedarf des „Sich-Sachkundig-Machens“ (Wanner 2015, S. 55).

Normalität und Othering Als Normalität ist durchwegs die Norm der Zweigeschlechtlichkeit gesetzt. Inter* wird als anders konstruiert, dies teilweise ganz offenbar, wenn etwa in einem Online-Artikel von den „anderen“ (Supp und Brandt 2007) die Rede ist (Wanner 2015, S. 40f), häufig aber subtiler. So sind im einleitenden Teil der Befragung von Inter* Personen (Bora 2012, S. 40) („Allgemeine Angaben“) vier von fünf Items als freistehende Substantivgruppen formuliert („Alter“, „Geschlecht gemäß Personenstandsrecht“, „Geschlecht, dem Sie sich selbst zuordnen“ und „Letzte gestellte medizinische Diagnose oder Diagnosen“ (ebd.)). Grammatikalisch implizieren diese Items die Möglichkeit von Ordnung, inhaltlich wäre die `Normalität der Zweigeschlechtlichkeit` denkbar. Erst jenes fünfte Item, das nach „Intersexualität“ fragt, stört als vollständige Phrase die Ordnung: „In welchem Alter haben sie von Ihrer Intersexualität erfahren?“ (ebd.). Die Analyse zeigt, dass Inter* als sanders`, gar als zu korrigierender Zustand, konstruiert ist - dies teilweise offensichtlich. So ist im OnlineDiskurs mitunter von „Abweichung“, „gestört“, „fehlerhaft“ (Fux 2010) die Rede.

\section{Trans*}

Trans* Personen wurden und werden wissenschaftlich konzeptioniert, was mit der Einführung einer psychiatrischen Diagnose einhergeht, deren Statthalter*innen (Psychotherapeut*innen, Mediziner*innen) die Möglichkeiten und die Finanzierung chirurgischer und hormoneller Eingriffe ganz im Sinne der Definitionsmacht verwalten. In der neuesten Auflage des durch die American Psychiatric Association herausgegebene Diagnostic and Statistical Manual of Mental Disorders (DSM) wird Trans* durch die Geschlechtsdysphorie als Inkongruenz zwischen dem bei der Geburt zugewiesenen Geschlecht und der Geschlechtsidentität klassifiziert. Solche Texte sind ein Zahnrad in der „diskursiven Maschinerie“ (Borba 2019, S. 25), die Trans* Personen als spezifische Subjekttypen in medizinischen und psychologischen Wissenssystemen identifiziert. Die Zweischneidigkeit einer Diagnose zeigt sich daran, dass sich einerseits Zugänge $\mathrm{zu}$ Kostenerstattung, etwa für Hormontherapie und Operationen, öffnen und eine Diagnose als Ausdruck eines möglichen hohen Leidensdrucks wahrgenommen werden kann. Gleichzeitig werden mit der Diagnose nicht-konforme Geschlechtsidentitäten pathologisiert und eigene verkörperte $\mathrm{Ge}$ schlechtlichkeit wird in institutionalisierte, gesell- schaftskonform glattpolierte und fremdbestimmende Entscheidungsstrukturen (unauflöslich) verflochten. Für die Arbeit mit Trans* Personen können sich Psychotherapeut*innen an internationalen Richtlinien ${ }^{10}$ orientieren, wobei es sich dabei vorwiegend um Konsensuserklärungen von sogenannten ‘Expert*innen ohne Einbezug der Perspektiven von Trans* Personen handelt. Dies erscheint in Bezug auf die oben ausgeführten Topoi, besonders die Abwertung von Erfahrungswissen, nicht überraschend: So existieren wenige Studien, die auf Erfahrungen von Trans* und Inter* Personen als TiAnP fokussieren - einige zentrale Elemente dennoch gefundener werden in der Folge vor der Hintergrundfolie obiger Ergebnisse diskutiert.

\section{Diskursives (Ra)umgestalten in der psychotherapeutischen Praxis}

Mizock und Lundquist (2016) haben in ihrer Studie Fehltritte herausgearbeitet, die von Trans* und GNC Personen in der Psychotherapie wahrgenommen werden. Diese stellen wir verknüpft mit zentralen Erkenntnissen zum Inter* Diskurs vor und geben anschließend praxisrelevante Empfehlungen.

Rolle als Wissensvermittelnde Als eine grundlegende negative Erfahrung beschreiben Trans* und GNC, dass Psychotherapeut*innen bei Themen, die sich auf die Geschlechtsidentität beziehen, stark auf die >Bildungsarbeit und Informationsvermittlung seitens ihres Gegenübers angewiesen sind (Mizock und Lundquist 2016, S. 148). Wenn auch der Zugang vielfach vertreten wird, Personen aus sogenannten marginalisierten Kontexten in der psychotherapeutischen Beziehung mit Neugier und Naivität zu begegnen, zeigen Mizock und Lundquist (2016), dass Trans* und GNC Personen ihre Rolle als Wissensvermittelnde mit Kompetenz- und Ausbildungslücken von Psychotherapeut*innen und großen Belastungen verbinden.

Gender Repairing Trans* und GNC erleben häufig, dass die Geschlechtsidentität in der Psychotherapie als zu behebendes Problem behandelt wird. So kommen Mizock und Lundquist (2016) zu ähnlichen Befunden wie sich in den Ergebnissen, die zum Inter* Diskurs als „Problemlösung ohne menschliches $\mathrm{Zu}$ tun“ beschrieben werden, zeigt.

Gender Avoidance Genderthemen und -aspekte werden völlig vermieden und kommen in der Psychotherapie nicht ausreichend zur Sprache. Eine Begründung leitet sich aus dem zu Inter* diskutierten Othering ab: Die Vermeidung vonseiten Psychothera-

10 Standards of Care der World Professional Association for Transgender Health (WPATH 2012) oder den Guidelines for Psychological Practice with Transgender and Gender Nonconforming People der American Psychological Association (APA 2015). 
peut*innen steht dann in Verbindung mit fehlendem (Erfahrungs-)Wissen, dass und inwiefern Genderaspekte Auswirkungen auf `mental health` haben.

Gender-Pathologizing Der Inter* Diskurs ist geprägt von der Konstruktion klarer Grenzen entlang von `Normalität` und >Abweichung`. Auch Mizock und Lundquist (2016) zeigen, dass die Anwendung restriktiver Vorstellungen von Geschlecht zur Folge hat, dass TiAn Trans* und GNC Personen wahrnehmen, in eben solche gepresst zu werden. Eine weitere Auswirkung von Gender-Pathologizing ist, dass Psychotherapeut*innen erlebte Erfahrungen lediglich als Symptome einer zu behandelnden, für alle Probleme verantwortlichen $`$ Krankheit betrachten.

Gender-Generalization Therapeut*innen, die nur eine (wortwörtlich) begrenzte Vorstellung von Gender haben, neigen dazu, die Themen und Probleme von Trans* und GNC Personen zu generalisieren und aus dem heraus richtige und falsche Wege zu suggerieren. Gender-Generalization begrenzt auch die Möglichkeiten - wie eine Schranke - Erfahrungen von TiAnP weitreichend $\mathrm{zu}$ erforschen, zu kommunizieren und Konflikthaftes aufzulösen.

Gender Inflation Ein übertriebener Fokus auf Genderthemen hat gegenläufig zur Folge, dass in der Psychotherapie weitere wichtige Aspekte des Lebens unbeachtet bleiben und so zu falschen Annahmen über die Entstehungsursachen von Symptomen wie auch über den Behandlungsschwerpunkt führen. Die ungenügende Erforschung anderer Lebensbereiche resultiere nach Mizock und Lundquist (2016) in einer permanenten Vergegenständlichung der Rolle der Geschlechtsidentität in den Erfahrungen. Vielfach wurde in dem Zusammenhang von Trans* und GNC Personen das Gefühl geschildert, nicht als ganze Person betrachtet zu werden - dem ist gerade vor der Hintergrundfolie der Entpersonifizierung als Pathologisierungsstrategie besondere Aufmerksamkeit zu schenken. Gender Inflation resoniert mit dem Befund, dass dem Geschlecht nach wie vor eine zentrale gesellschaftliche Ordnungsfunktion zugedacht ist.

Gatekeeping Insbesondere im Transitionsprozess kommt es vor, dass Trans* und GNC die Rolle der Psychotherapeut*in als ausschließlich auf den Kontrollzugang $\mathrm{zu}$ medizinischen Ressourcen konzentriert wahrnehmen. Diese Rolle wird durch Trans* und GNC Personen auch in Anlehnung an Singh und Burnes (2010) vor allem dann als problematisch wahrgenommen, wenn Psychotherapeut*innen als unkooperativ oder zu restriktiv erlebt werden. Eindeutig stehen diese Befunde in Einklang mit dem zum Inter* Diskurs beschriebenen Topos der medizinischen Definitionshoheit („die Ordnung, das sind die anderen“). Realität ist, dass Psychotherapeut*innen und Mediziner*innen letztlich über die (Möglichkei- ten) verkörperte(r) Geschlechtlichkeit bestimmen diese Rolle ist untrennbar mit der Verantwortung verbunden, sie mit höchstem Feingefühl und unter permanenter Hinterfragung eigener Narrative auszuüben. Die Spannung, dass schon die Ausübung psychotherapeutischer Praxis an sich eine Reproduktion der Machtstruktur darstellt, bleibt dabei erhalten.

Als erste praktische Konsequenz leiten wir aus den besprochenen Ergebnissen die Notwendigkeit ab, Erfahrungswissen anzuerkennen und Inter* und Trans* Personen als Sprecher*innen und Expert*innen ernst zu nehmen und zu stärken. Spezifische Mikroaggressionen, denen Trans* und Inter* Personen ausgesetzt sind (Chang und Chung 2015) können zu Erwartungen von Ablehnung oder Diskriminierung in der psychotherapeutischen Beziehung führen. Es ist dabei zentral, gesellschaftliche Diskriminierungserfahrung als solche wahrzunehmen, nicht $\mathrm{zu}$ verharmlosen und die je eigene Definitionsmacht hinsichtlich der Geschlechtlichkeit zu respektieren; dies schließt auch die Frage nach und die Verwendung von selbstgewählten Pronomen mit ein. Gleichzeitig, und hier ist hohe Sensibilität gefordert, soll der therapeutische Raum ermöglichen, eigene Deutungs- und Wahrnehmungsmuster in einem möglichst sicheren Rahmen zu reflektieren. Dafür ist die Auseinandersetzung mit und die Reflexion von Geschlechtlichkeit aufseiten der Therapeut*innen wesentlich (McCann und Sharek 2016). Poteat und Kolleg*innen (2013) weisen anhand von Tiefeninterviews mit TiAn Trans* Personen und klinischem Personal aus dem US-amerikanischen Gesundheitsbereich darauf hin, dass ein diesbezüglicher Mangel die Beziehungsgestaltung so stark beeinflussen kann, dass sich die individuelle Situation von TiAnP verschlechtert, die Behandlungszufriedenheit verringert und die zukünftige Annahme von Unterstützungsangeboten nachhaltig beeinträchtigt wird. Zudem stellen White Hughto und Kollegen* (2015) dar, wie Stigmata gegenüber Trans* Personen auf individuellen, interpersonalen und institutionellen Ebenen wirken und argumentieren vor dem Hintergrund von Interventionsmöglichkeiten und anknüpfend an die Befunde von Poteat et al. für eine verstärkte Schwerpunktsetzung im Rahmen der Ausbildung (S. 227). Für die psychotherapeutische Arbeit haben wir folgende Orientierungslinien formuliert:

1. Erwerb von Wissen, z.B. Inanspruchnahme und (mitunter auch finanzielle) Wertschätzung von Beratung durch (zumeist unbezahlt arbeitende) Selbstvertreter*innen und Aktivist*innen; begleitende Supervision.

2. Hegemoniale Diskurse zu Geschlechtern, Sexualitäten und deren Zusammenhänge mit Machtverhältnissen kennen und reflektieren, sodass sie den psychotherapeutischen Raum weniger stark beherrschen und dennoch als Realität sichtbar sind; Kulturelle/gesellschaftliche (Selbst-)Stigmatisierungen 
von Geschlecht erkennen und unterbrechen: Nach Hakeem (2010) fördert dies nicht nur ein besseres Verständnis der TiAnP, sondern stellt auch einen sozialen, politischen und kulturellen Beitrag dar, um auf Veränderungen in der gesellschaftlichen Grundmatrix hinzuarbeiten.

3. Heranziehung intersektionaler Ansätze, die neben gender Kategorien wie race, class, dis/ability und weitere mit einbeziehen (Duong 2012): Da psychotherapeutische Zugänge im überwiegenden Maße auf heteronormativen, monokulturellen Annahmen basieren, ist es wichtig, multidimensionale Facetten sozialer und psychischer Realitäten mitzubedenken; die Reflexion historisch gewachsener Privilegien und Macht erscheint dafür unerlässlich.

Geschlechtliche Dimensionen und damit verbundene Diskurse durchwandern alle gesellschaftlichen - und damit auch psychischen Welten. Wir haben darüber nachgedacht, was dies für psychotherapeutische Räume bedeuten kann - dies aus der Perspektive weißer*, temporarily able-bodied Akademiker*innen und angehender Psychotherapeut*innen als Autor*innen, die sich als Trans* jenseits binärer Geschlechtszuschreibungen bzw. als queere Frau* selbst bezeichnen. Wir haben in diesem Artikel Trans*, Inter* und GNC aufseiten der TiAnP fokussiert. Dabei haben wir uns auf Befunde gestützt, die das Erfahrungswissen von Trans* und GNC Personen ins Zentrum stellen. Fragen danach, wie Trans*, Inter* und GNC Personen auf Therapeut*innenseite den psychotherapeutischen Raum erleben und gestalten, sind ebenso spannend. Lautstark kritisieren wir, dass die Wege zur psychotherapeutischen Praxis für Inter*, Trans* und GNC Personen durch massiv ausgrenzende Zugangsbarrieren erschwert bis hin zu versperrt sind.

Wir imaginieren psychotherapeutische Räume, die freier sind: Um sich selbst in Erfahrung zu bringen/zu singen; um weniger Schranke und weniger Quadra(n)t sein $\mathrm{zu}$ müssen.

Interessenkonflikt L. Wanner und F.L. Landsteiner geben an, dass kein Interessenkonflikt besteht.

Open Access Dieser Artikel wird unter der Creative Commons Namensnennung 4.0 International Lizenz (http:// creativecommons.org/licenses/by/4.0/deed.de) veröffentlicht, welche die Nutzung, Vervielfältigung, Bearbeitung, Verbreitung und Wiedergabe in jeglichem Medium und Format erlaubt, sofern Sie den/die ursprünglichen Autor(en) und die Quelle ordnungsgemäß nennen, einen Link zur Creative Commons Lizenz beifügen und angeben, ob Änderungen vorgenommen wurden.

\section{Literatur}

American Psychological Association. (2015). Guidelines for psychological practice with transgender and gender nonconforming people. American Psychologist, 70(9), 832-864.
Anohni (2009). Epilepsy is dancing [Aufgen. v. Antony and the Johnsons]. The crying light. London: Rough Trade Records.

Austin, A., Craig, S., \&Alessi, E. (2016). Affirmative cognitivebehavior therapy with Transgender and gender nonconforming adults. Psychiatric Clinics of North America, 40(1), 141-156.

Bora, A. (2012). Zur Situation intersexueller Menschen. Bericht über die Online-Umfrage des Deutschen Ethikrates. http://www.ethikrat.org/dateien/pdf/bora-zursituation-intersexueller-menschen.pdf. Zugegriffen: 11. Febr. 2015.

Borba, R. (2019). The interactional making of a "true transsexual": Language and (dis)identification in trans-specific healthcare. International Journal of the Sociology of Language, 256, 21-55.

Chang, T. K., \&Chung, Y. B. (2015). Transgender microaggressions: Complexity of the heterogeneity of transgender identities. Journal of LGBT Issues in Counseling, 9, 217-234.

Corbett, K. (2009). Boyhoods. Rethinking Masculinities. New Haven \& London: Yale University Press.

Deutscher Ethikrat (2011c). Audioprotokoll Anhörung Intersexualität. http://www.ethikrat.org/veranstaltungen/ anhoerungen/intersexualitaet. Zugegriffen: 11. Febr. 2015.

Deutscher Ethikrat (2011b). Simultanmitschrift. Öffentliche Anhörung und Dialog. http://www.ethikrat.org/ dateien/pdf/anhoerung-intersexualitaet-2011-06-08simultanmitschrift.pdf.Zugegriffen: 11. Febr. 2015.

Deutscher Ethikrat (2011a). Schriftliche Stellungnahmen der Sachverständigen zur Intersexualität. http://www. ethikrat.org/sachverstaendigenbefragungintersexualitaet.Zugegriffen: 11. Febr.2015.

Deutscher Ethikrat (2012). Intersexualität. http://www. ethikrat.org/dateien/pdf/stellungnahmeintersexualitaet.pdf.Zugegriffen: 10. Febr. 2015.

Distiller, N. (2011). Fixing gender: lesbian mothers and the oedipus complex. Lanham: Fairleigh Dickinson Univ. Press/The Rowman \& Littlefield.

Duong, K. (2012). What does queer theory teach us about intersectionality? Politics \& Gender, 8, 370-386.

Europäisches Parlament (2019). European Parliament resolution on the rights of intersex people. http://www. europarl.europa.eu/doceo/document/B-8-2019-0101_ EN.pdf.Zugegriffen: 16. Febr. 2019.

Foucault, M. (2012). Das wahre Geschlecht. In W. Schäffner \& J. Vogl (Hrsg.), Über Hermaphrodismus. Der Fall Barbin (S.7-18). Frankfurta. M.: Suhrkamp.

Fraser, L. (2009). Depth psychotherapy with transgender people. Sexual and Relationship Therapy, 24(2), 126-142.

Fux, C. (2010). Intersexualität. In der Grauzone der Geschlechter. http://www.focus.de/gesundheit/ratgeber/ sexualitaet/tid-13548/intersexualitaet-in-der-grauzoneder-geschlechter_aid_376653.html. Zugegriffen: 9. Febr. 2015.

Hakeem, A. (2010). Deconstructing gender in trans-gender identities. Group Analysis, 43(2), 141-154.

Jäger, S. (2009). Kritische Diskursanalyse. Eine Einführung (5. Aufl.). Münster: Unrast.

Knutson, D., \& Koch, J.M. (2018). Person-centered therapy as applied to work with transgender and gender diverse clients. Journal of Humanistic Psychology, 2019 https:// doi.org/10.1177/0022167818791082.

Mallory, A., Brown, J., Conner, S., \& Henry, U. (2017). Finding what works: new clinicians' use of standards of care with transgender clients. The American Journal of Family Therapy, 45(1), 27-36. 
McCann, E., \& Sharek, D. (2016). Mental health needs of people who identify as transgender: a review of the literature. Archives of Psychiatric Nursing, 30(2), 280-285.

Mizock, L., \& Lundquist, C. (2016). Missteps in psychotherapy with transgender clients: promoting gender sensitivity in counseling and psychological practice. Psychology of Sexual Orientation and Gender Diversity, 3(2), 148-155.

Plattform Intersex (2019). Presseaussendung: EU-Resolution $\mathrm{zu}$ Inter. http://www.plattform-intersex.at/?

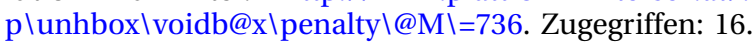
Febr. 2019

Poteat, T., et al. (2013). Managing uncertainty: a grounded theory of stigma in transgender health care encounters. Social Science \& Medicine, 84,22-29.

Puckett, J.A., \& Levitt, H. M. (2015). Internalized stigma within sexual and gender minorities: change strategies and clinical implications. Journal of LGBT Issues in Counseling, 9(4), 329-349.

Singh, A.A., \& Burnes, T. R. (2010). Shifting the counselor role from gatekeeping to advocacy. Journal of LGBT Issues in Counseling, 4(3-4), 241-255.

Siročić, Z. (2015). Gender: gendering of categories. In J.D. Wright (Hrsg.), International encyclopedia of the social \& behavioral sciences (S.788-794). Oxford: Pergamon.

Slunecko, T. (2012). Zur Kritik der Zuschauerontologie der Psychoanalyse - vorbereitende Arbeiten. In G. Gödde \& M.
Buchholz (Hrsg.), Der Besen, mit dem die Hexe fliegt. Wissenschaft und Therapeutik des Unbewussten (S.563-582). Gießen:Psychosozial-Verlag.

Supp, B., \& Brandt, A. (2007). Und Gott schuf das dritte Geschlecht. http://www.spiegel.de/spiegel/print/d54002246.html.Zugegriffen:9. Febr. 2015.

Völling, C. (2010). Ich war Mann und Frau. Mein Leben als Intersexuelle. Köln: Fackelträger.

Voß, H.-J. (2012). Intersexualität - Intersex. Eine Intervention. Münster: Unrast.

Wanner, L. (2015). Hier bin ich Geschlecht, hier darf ich sein. Diskursanalyse zur Inter*-Debatte des Deutschen Ethikrates. Unveröff. Diplomarbeit an der UniversitätWien

White Hughto, J., Reisner, S. L. \& Pachankis, J. (2015). Transgender Stigma and Health: A Critical Review of Stigma Determinants, Mechanisms, and Interventions. Social Science \& Medicine, 147,222-231.

World Professional Association for Transgender Health. (2012). Standards of Care for the Health of Transsexual, Transgender, and Gender Nonconforming People (7. Version). https://www.wpath.org/publications/soc. Zugegriffen:20. Mai2019.

Hinweis des Verlags Der Verlag bleibt in Hinblick auf geografische Zuordnungen und Gebietsbezeichnungen in veröffentlichten Karten und Institutsadressen neutral. 